\title{
Compact microwave ion source for extremely low energy ion irradiation system
}

$\operatorname{AUTHOR}(S):$

Gotoh, Y; Kubo, H; Tsuji, H; Ishikawa, J

\section{CITATION:}

Gotoh, Y ... [et al]. Compact microwave ion source for extremely low energy ion irradiation system. REVIEW OF SCIENTIFIC INSTRUMENTS 2000, 71(2): 1160-1162

\section{ISSUE DATE:}

2000-02

URL:

http://hdl.handle.net/2433/39803

\section{RIGHT:}

Copyright 2000 American Institute of Physics. This article may be downloaded for personal use only. Any other use requires prior permission of the author and the American Institute of Physics. 


\title{
Compact microwave ion source for extremely low energy ion irradiation system
}

\author{
Y. Gotoh, ${ }^{a)}$ H. Kubo, H. Tsuji, and J. Ishikawa \\ Department of Electronic Science and Engineering, Kyoto University, Yoshida-honmachi, Sakyo-ku, \\ Kyoto 606-8501, Japan
}

(Presented on 9 September 1999)

\begin{abstract}
The performance of a compact microwave ion source developed for extremely low energy ion extraction was measured. The ion source was modified to be fitted for the ion extraction at the voltage lower than $100 \mathrm{~V}$. The current-voltage characteristics, mass spectrum, absolute ion energy, and energy spread of argon ion beam were estimated with a sector magnet. The results showed that the mass spectrum showed a clear separation of singly charged ions and doubly charged ions even at the extraction voltage of $4 \mathrm{~V}$. Mass separated $\mathrm{Ar}^{+}$current of $0.5 \mathrm{nA}$ at $4 \mathrm{~V}$ extraction was obtained. An excess ion energy due to presence of plasma potential was $14 \mathrm{eV}$ at the pressure of $5 \times 10^{-3} \mathrm{~Pa}$, and $66 \mathrm{eV}$ at $9 \times 10^{-4} \mathrm{~Pa}$. Energy spread was narrower than $12 \mathrm{eV}$, from rough estimate of the mass spectrum. These results summarize that the present ion source can be used for extremely low energy ion irradiation system. (c) 2000 American Institute of Physics.
\end{abstract}

[S0034-6748(00)54402-5]

\section{INTRODUCTION}

It is well known that presence of energetic particles during thin film deposition influences the resulting film property significantly. ${ }^{1,2}$ The effective ion energy is almost a hundred electron volts for ion beam deposition, and below this energy, for example, atomic bonding of carbon film dramatically changes. ${ }^{3}$ However, due to uncertainty in theory of ionsolid interaction at such a low energy, the reason why this ion energy is appropriate, has not been clarified as yet. In order to clarify the mechanism of thin film formation with energetic particles, it is necessary to observe the ion-solid interaction for one ion by one ion directly. From this point of view, we have been investigating the ion irradiated solid surface with a scanning tunneling microscope. ${ }^{4,5}$ In order to perform the research, a reliable ion source which provides sufficient amount of low energy, monochromatic ions, is desired. We should avoid accel-decel type transportation because elimination of neutralized high energy ions is not easy. Thus, direct extraction at irradiating energy is necessary. Previously, we have reported development of a compact microwave ion source for low energy application. ${ }^{6}$ This ion source was a modified version of the compact microwave ion source developed earlier. ${ }^{7}$ In this study, we examined the feasibility of this modified ion source as that for low energy ion irradiation system. The ion extraction system was further modified to be fitted for extraction at extremely low voltage. The ion beam extracted from the ion source was mass analyzed and fundamental study on the quality of the low energy ion beam was performed.

\section{EXPERIMENTAL SETUP}

Figure 1 shows the schematic diagram of the present ion source. The ion extraction system consisted of two elec-

${ }^{a)}$ Electronic mail: ygotoh@kuee.kyoto-u.ac.jp trodes, plasma electrode and extraction electrode. In order to achieve better ion optics at the extraction region, the thicknesses of these electrodes were $0.3 \mathrm{~mm}$ and the gap between the plasma electrode and the extraction electrode was set to be $0.35 \mathrm{~mm}$. Each electrode had an tapered aperture with a diameter of $0.5 \mathrm{~mm}$ for ion extraction. Computer simulation of this extraction system showed that optimum extraction voltage is higher than $100 \mathrm{~V}$. But thinner electrode or narrower gap may cause distortion of electrodes or unexpected discharge, thus, in this study, earlier geometry was used.

The test bench was equipped with a $30^{\circ}$ deflection sector magnet with a radius of curvature of $300 \mathrm{~mm}$. The ion beam was limited by an aperture with a diameter of $4 \mathrm{~mm}$ which located at $11 \mathrm{~cm}$ below the ion source. The analyzer magnet was set at $16 \mathrm{~cm}$ below this aperture. A collector with the width of $2 \mathrm{~mm}$ and the effective area of $0.8 \mathrm{~cm}^{2}$ was settled at $13.5 \mathrm{~cm}$ below the exit of the mass separator. The massseparated ion current was monitored with a pico-ammeter. The source chamber was evacuated by a turbomolecular pump with the nominal pumping speed of $80 \mathrm{~L} \mathrm{~s}^{-1}$. The residual gas pressure just above the pump was $<3$ $\times 10^{-4} \mathrm{~Pa}$ and most of the experiments were performed at the argon gas pressure $\left(P_{\mathrm{Ar}}\right)$ of $5 \times 10^{-3} \mathrm{~Pa}$.

\section{CHARACTERISTICS OF ION SOURCE}

\section{A. Current-voltage characteristics}

The current-voltage $(I-V)$ characteristics before mass separation was performed with an ion limiter as an ion collector. The ion current at the extraction voltage $\left(V_{\text {ext }}\right)$ of 100 $\mathrm{V}$ was $1 \mu \mathrm{A}$, and it decreased with decreasing the extraction voltage. At the extraction voltage below $20 \mathrm{~V}$, the collector current became negative. This would be due to high energy electrons involved in the ion beam. Presence of ions even at a low $V_{\text {ext }}$ of several volts was confirmed in the following measurements of mass spectrum. 


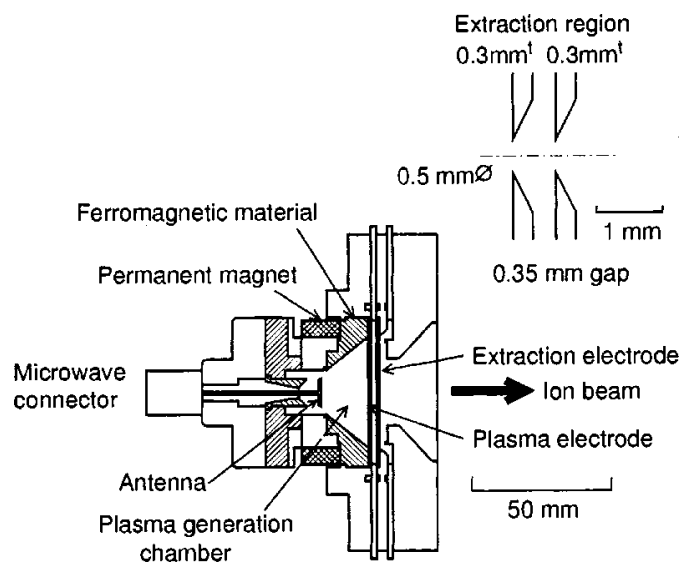

FIG. 1. Schematic diagram of the ion source.

\section{B. Mass spectrum}

Figures 2(a)-2(d) show the typical mass spectra of argon ion beams at various acceleration voltage. At $V_{\text {ext }}$ of $10 \mathrm{~V}$, $\mathrm{Ar}^{+}$and $\mathrm{Ar}^{2+}$ could be completely separated. Even at $V_{\text {ext }}$ of $4 \mathrm{~V}$, we can distinguish between $\mathrm{Ar}^{+}$and $\mathrm{Ar}^{2+}$. However, at $V_{\text {ext }}$ of $3 \mathrm{~V}$, these two peaks overlapped.

\section{C. $I-V$ characteristics after mass separation}

Figure 3 shows the typical $I-V$ characteristics after mass separation. The $\mathrm{Ar}^{+}$current after mass separation was almost $1 \mathrm{nA}$ at $V_{\text {ext }}$ of $100 \mathrm{~V}$. The current decreased with decreasing $V_{\text {ext }}$ but turned to increase below $V_{\text {ext }}$ of $10 \mathrm{~V}$. The reason for this is not clear at present, but the followings are possible candidates: (a) immersion of plasma to the extraction region improved the ion extraction optics, and/or (b) presence of electrons reduced the space charge at the ion sheath. Irrespective of $V_{\text {ext }}$, yield of $\mathrm{Ar}^{2+}$ was approximately $10 \%$ as compared with the yield of $\mathrm{Ar}^{+}$.

Since the corrector area was $0.8 \mathrm{~cm}^{2}$, the ion currents of 0.1 and $1 \mathrm{nA}$ correspond to the ion current density of 0.13 and $1.3 \mathrm{nA} \mathrm{cm}^{-2}$. These ion current densities correspond to 0.08 and 0.8 ions per $100 \mathrm{~nm} \times 100 \mathrm{~nm}$, per second. We are observing the ion irradiated surface with the dose of approxi-
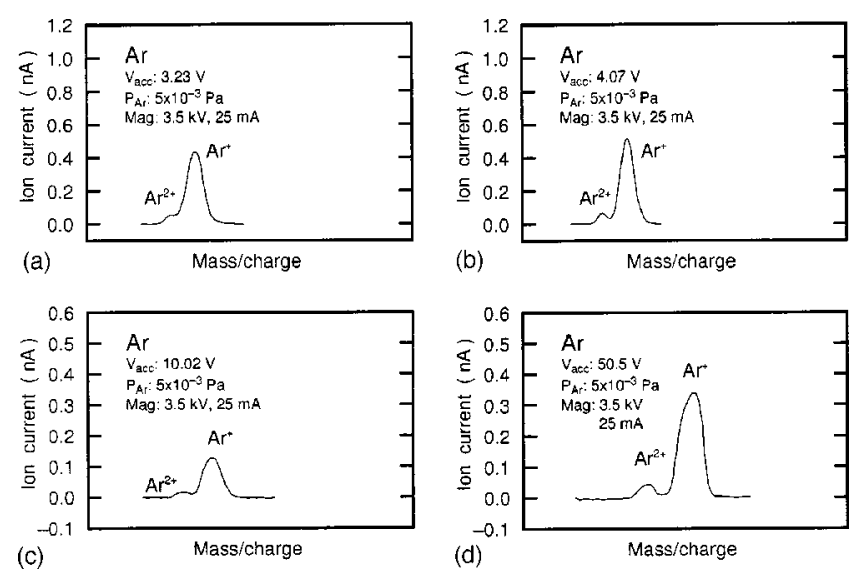

FIG. 2. Typical mass spectra of Ar ion beams extracted at various extraction voltages.

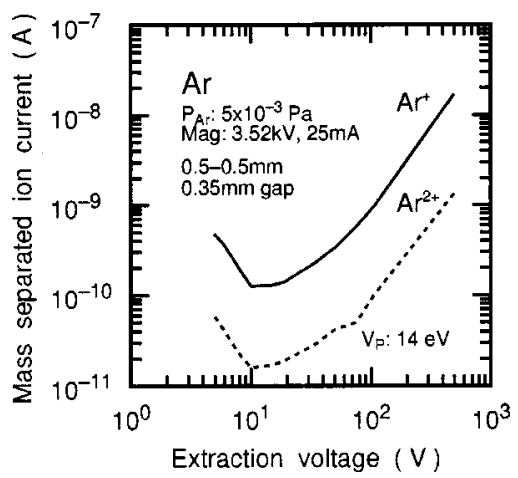

FIG. 3. Mass separated $\mathrm{Ar}^{+}$and $\mathrm{Ar}^{2+}$ currents as a function of extraction voltage.

mately a hundred ions per $100 \mathrm{~nm} \times 100 \mathrm{~nm}$, so required irradiation time is almost 21 to $2.1 \mathrm{~min}$. This irradiation time is appropriate to control the dose.

\section{Estimation of ion energy}

For the ion beam extracted from plasma has an excess energy due to presence of plasma potential. This excess energy is not neglected for low energy application. Ion energy was estimated from the dependence of coil current necessary to pass $\mathrm{Ar}^{+}$and $\mathrm{Ar}^{2+}$ ions on the extraction voltage. The experiments were performed at two different gas pressures: $5 \times 10^{-3}$ and $9 \times 10^{-4} \mathrm{~Pa}$. The magnetic field, which was represented by the peak position in the mass spectrum, should be proportional to the square root of ion energy

$$
x \propto I \propto H \propto \sqrt{\frac{2 m\left(V_{\mathrm{ext}}+V_{p}\right)}{e} .}
$$

Thus, $x^{2}$ is proportional to $V_{\mathrm{ext}}+V_{p}$. The plasma potential can be known from the diagram of $x^{2}-V_{\text {ext }}$ with its intercept. Figure 4 shows the typical example of the relation between $V_{\text {ext }}$ and $x^{2}$ of $\mathrm{Ar}^{+}$and $\mathrm{Ar}^{2+}$. Open circles and squares indicate the characteristics of $\mathrm{Ar}^{2+}$ and $\mathrm{Ar}^{+}$at $P_{\mathrm{Ar}}$ of 5 $\times 10^{-3} \mathrm{~Pa}$, and solid circles and squares indicate those at $P_{\mathrm{Ar}}$ of $9 \times 10^{-4} \mathrm{~Pa}$. The intercepts at the ordinate are 14 and $66 \mathrm{~V}$, thus, the plasma potentials were estimated to be 14 and $66 \mathrm{eV}$, respectively.

The higher plasma potential at lower gas pressure would be due to higher electron temperature in the plasma. However, ratio of $\mathrm{Ar}^{2+} / \mathrm{Ar}^{+}$was decreased for the case of lower

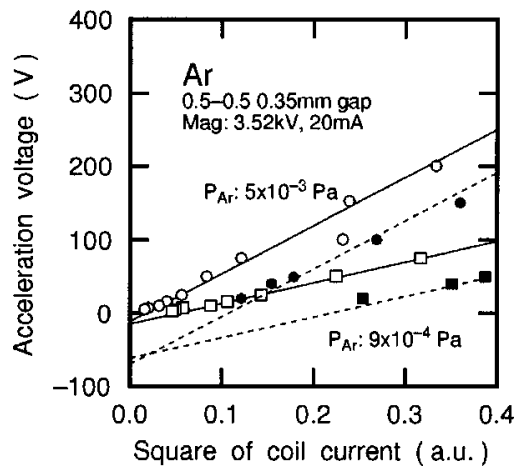

FIG. 4. Relation between the coil current necessary to pass $\mathrm{Ar}^{+}$and $\mathrm{Ar}^{2+}$ ions and extraction voltage. 
gas pressure. The details on the plasma density and the electron temperature should be investigated in future. At least, the results indicated that precise control of gas pressure is necessary.

\section{E. Estimation of energy spread}

From these results, we can very roughly estimate the energy spread of the argon ion beam. The absolute ion energy for $\mathrm{Ar}^{+}$at $V_{\text {ext }}$ of $4 \mathrm{~V}$ is found to be $18 \mathrm{eV}$. If we neglect the width of the ion collector, which determines the resolution function, maximum energy spread can be estimated in the following manner. Condition for coincidence of the peak position of $\mathrm{Ar}^{+}$with the lowest energy and that of $\mathrm{Ar}^{2+}$ with the highest energy should be

$$
H \propto \frac{1}{r} \sqrt{\frac{2 m(V-\Delta V)}{e}}=\frac{1}{r} \sqrt{\frac{2 m(V+\Delta V)}{2 e}},
$$

where $\Delta V$ is the half of the energy spread. From this relation $\Delta V$ is at most $6 \mathrm{eV}$. Thus, the energy spread would be less than $12 \mathrm{eV}$. Considering that the finite entrance aperture, the energy spread would be much more narrower than the above value. The reason for poor resolution at $V_{\text {ext }}$ of $3 \mathrm{~V}$ would be attributed to the fact that protruding the plasma toward extraction electrode due to lower electric field caused diver- gence of the beam, resulting in broader peak after mass separation. From this result, it was found that the ion beam with narrow energy spread could be obtained.

\section{CONCLUSION}

A compact microwave ion source for extremely low energy ion irradiation system was developed and the performance was evaluated. The results indicated that a low energy ion beam with a sufficient current and a low energy spread could be obtained, but the ion energy depended strongly on the gas pressure. Precise control of the gas pressure would be necessary. To identify the energy spread, detailed measurement of energy distribution will be necessary.

${ }^{1}$ J. A. Thornton and D. W. Hoffman, Thin Solid Films 171, 5 (1989).

${ }^{2}$ S. M. Rossnagel and J. J. Cuomo, Vacuum 38, 73 (1988).

${ }^{3}$ J. Ishikawa, Y. Takeiri, K. Ogawa, and T. Takagi, J. Appl. Phys. 61, 2509 (1987).

${ }^{4}$ J. Ishikawa, H. Tsuji, K. Kameyama, S. Shimada, and Y. Gotoh, Appl. Surf. Sci. 100/101, 370 (1996).

${ }^{5}$ Y. Gotoh, H. Kubo, Y. Yamauchi, H. Tsuji, and J. Ishikawa, Abstract of 14th International Vacuum Congress, 10th International Conference on Solid Surfaces, 5th International Conference on Nanometer-scale Science and Technology, 10th International Conference on Quantitative Surface Analysis, Birmingham, UK, August 31-September 4 (1998) p. 155.

${ }^{6}$ Y. Gotoh, Y. Fujimori, H. Kubo, H. Tsuji, and J. Ishikawa, Rev. Sci. Instrum. 69, 887 (1998).

${ }^{7}$ J. Ishikawa, Y. Takeiri, and T. Takagi, Rev. Sci. Instrum. 55, 449 (1984). 